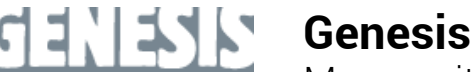

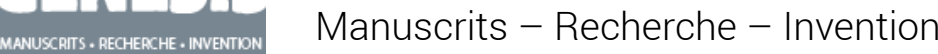

$45 \mid 2017$

Hugo

\section{En marchant, en dessinant}

L'inscription du corps en mouvement dans la pratique graphique de Victor Hugo

\section{Delphine Gleizes}

\section{(2) OpenEdition}

1 Journals

Édition électronique

URL : http://journals.openedition.org/genesis/2915

DOI : $10.4000 /$ genesis.2915

ISSN : 2268-1590

Éditeur :

Presses universitaires de Paris Sorbonne (PUPS), Société internationale de génétique artistique littéraire et scientifique (SIGALES)

\section{Édition imprimée}

Date de publication : 5 décembre 2017

Pagination : 83-98

ISBN : 979-10-231-0580-3

ISSN : $1167-5101$

\section{Référence électronique}

Delphine Gleizes, « En marchant, en dessinant », Genesis [En ligne], 45 | 2017, mis en ligne le 15 décembre 2018, consulté le 11 janvier 2021. URL : http://journals.openedition.org/genesis/2915 ; DOl : https://doi.org/10.4000/genesis.2915 


\section{En marchant, en dessinant L'inscription du corps en mouvement dans la pratique graphique de Victor Hugo}

Delphine Gleizes

$\mathrm{D}$ ans le premier article de la série «Réflexions sur quelques-uns de mes contemporains», Baudelaire rendait en 1861 un hommage appuyé au grand absent des Lettres françaises :

Depuis bien des années déjà Victor Hugo n'est plus parmi nous. Je me souviens d'un temps où sa figure était une des plus rencontrées parmi la foule; et bien des fois je me suis demandé, en le voyant si souvent apparaître dans la turbulence des fêtes ou dans le silence des lieux solitaires, comment il pouvait concilier les nécessités de son travail assidu avec ce goût sublime, mais dangereux, des promenades et des rêveries. Cette apparente contradiction est évidemment le résultat d'une existence bien réglée et d'une forte constitution spirituelle qui lui permet de travailler en marchant, ou plutôt de ne pouvoir marcher qu'en travaillant ${ }^{1}$.

La marche, pour l'écrivain, le porte bien au-delà d'un exercice romantique auquel pourtant il se livra régulièrement. Célestin Nanteuil ne le conforta-t-il pas dans la pratique du carnet de voyage lors d'un périple en Normandie, en juin 1836, en compagnie de Juliette Drouet, en lui laissant son propre album de dessin et en l'incitant à achever de le remplir ${ }^{2}$ ? Hugo n'ignore rien non plus des codes des Voyages pittoresques alors fort en vogue - que l'on songe à l'entreprise de Taylor, Cailleux et Nodier ${ }^{3}$ - et dans la lignée desquels s'inscrivit Abel Hugo ${ }^{4}$. Le Rhin et l'ensemble des notes de voyage rédigées par Hugo en portent trace et l'écrivain eut lui-même le projet, abandonné, d'un Voyage poétique et pittoresque au Mont-Blanc et à la vallée de Chamouny ${ }^{5}$. Détour fantaisiste, curiosité d'antiquaire, excursion pittoresque, la marche chez Hugo est bien tout cela, mais elle ne se borne pas à ces pratiques. Baudelaire l'avait bien compris qui, tout en ébauchant la silhouette symbolique d'un éternel homo viator en butte aux vicissitudes de la destinée et aux rigueurs de l'exil, voyait dans la marche l'élément dynamique d'une genèse dont le pas du marcheur, dans son amplitude, serait comme le principe inchoatif. Constitutive d'un rapport au monde, la marche est pour Hugo parcours sensible et effort spirituel. Elle est l'occasion d'un arpentage qui permet de prendre la mesure des choses, d'en déchiffrer le poème. «Rêveur», «camarade», contemplateur, l'artiste prend pied dans le réel et l'enjambe tout à la fois dans un franchissement des apparences. La marche confère à la main qui écrit, mais aussi à la main qui dessine, une charge cinétique dont il faudrait tenter ici de cerner les différentes valeurs et variations.

\section{$*$}

Le voyage, pour l'écriture comme pour le dessin, constitue un biais majeur de création. Toute excursion, tout périple devient matière à imagination plastique ou matériau pour un «roman futur», ainsi que l'écrivait Hugo à son fils Charles à propos de son voyage sur l'île de Serk et de son projet d'écriture des Travailleurs de la mer 6 .

1. Charles Baudelaire, «Réflexions sur quelques-uns de mes contemporains. I - Victor Hugo » [Revue fantaisiste, 15 juin 1861, 9e livraison, p. 131]; Euvres complètes, éd. Claude Pichois, Paris, Gallimard, coll. «Bibliothèque de la Pléiade», [1976], 1985, t. II, p. 129.

2. Victor Hugo, Euvres complètes, édition chronologique publiée sous la direction de Jean Massin, Club français du livre, 1967-1970 [dorénavant CFL], t. XVIII, voyage de $1836, \mathrm{n}^{\circ}$ A-F pour les dessins de Nanteuil, $n^{\circ}$ 75-90 pour ceux de Hugo. Voir plus généralement la remarquable présentation de Pierre Georgel, «Les dessins de voyage de Victor Hugo » (ibid., t. V), à laquelle les présentes remarques renvoient plus d'une fois. 3. Voyages pittoresques et romantiques dans l'ancienne France, par MM. Ch. Nodier, J. Taylor et Alph. de Cailleux, Paris, Gide fils (A.-F. Lemaître), 1820-1878.

4. Abel Hugo, France pittoresque, ou Description pittoresque, topographique et statistique des départements et colonies de la France, 3 vol., Delloye, 1835.

5. CFL, t. II, p. 1501, 1539-1542 et 1653.

6. «Ce nonobstant, j'irai peut-être passer quelques jours à Serk pour prendre les notes du roman futur» (Victor Hugo à Charles Hugo, 14 mai 1859; ibid., t. X, p. 1303). 
Il s'agit d'une étape essentielle dans l'invention créatrice, que le dessin - croquis souvent ébauché en promenade apparaisse comme préliminaire à une composition artistique plus élaborée ou qu'il constitue une formule transitoire dont la matière sera ensuite redistribuée dans l'univers textuel, poétique ou romanesque. Victor Hugo emmagasine le réel, matériau premier de l'imaginaire. Baudelaire l'avait bien senti, qui rappelait dans une prosopopée mélancolique les techniques de l'écrivain :

Sans cesse, en tous lieux, sous la lumière du soleil, dans les flots de la foule, dans les sanctuaires de l'art, le long des bibliothèques poudreuses exposées au vent, Victor Hugo, pensif et calme, avait l'air de dire à la nature extérieure : «Entre bien dans mes yeux pour que je me souvienne de toi ${ }^{7}$.»

Le visible rencontré chemin faisant s'affirme par sa fécondité génétique, non pas seulement en ce qu'il offre une collection de fragments du sensible, mais parce que l'expérience de la marche l'a en quelque sorte d'ores et déjà configuré.

Un corps qui déambule organise l'espace autour de lui, ménage des perspectives, construit des points de vue. Dans ses carnets de voyage, Hugo note très fréquemment en regard du jour et de l'heure le point depuis lequel les croquis ont été réalisés. Parfois, la comparaison d'une ébauche réalisée en excursion et d'un dessin plus abouti permet d'apprécier la façon dont le point de vue du marcheur a pu peser sur la représentation. C'est le cas par exemple pour les dessins que Hugo consacre à la Souris, burg rhénan qu'il a «dessiné très exactement ${ }^{8}$ » comme il l'écrit à son fils Charles dans la lettre du 1er octobre 1840 qui accompagne l'envoi d'un beau dessin à l'encre (fig. 1). Une ébauche de la pittoresque bâtisse apparaît au folio $40 \mathrm{v}^{\circ}$ du manuscrit Naf 13444 en date du 16 septembre 1840 (fig. 2). Le trait, assez sommaire, est exécuté à la mine de plomb. L'effet de contre-plongée y est assez marqué, sans doute lié à la position du poète à flanc de colline. La comparaison d'ailleurs avec le dessin de la page suivante, $\mathrm{f}^{\circ} 41 \mathrm{v}^{\circ}$, renforce l'impression de mobilité du point de vue, d'autant plus que les deux croquis, si l'on en croit les notations portées par la main de Victor Hugo, ont été réalisés à une heure et quart d'intervalle ${ }^{9}$. Le dessin à l'encre adressé à Charles est en ce sens plus équilibré : la ligne d'horizon est remontée, le point d'observation est comme reculé, ce qui confère à la représentation une plus grande stabilité et un statut plus marqué de «tableau». Mais le dessin conserve néanmoins de la première esquisse son caractère imposant lié à la présence d'une haute tour : cette dernière était déformée par la contre-plongée; elle est dans le dessin pour Charles «dramatisée» par l'accentuation de sa hauteur et le contraste des teintes sombres sur le ciel clair.

L'ensemble des dessins, pris en série, manifeste de manière sous-jacente un engagement du corps. Un adjectif revient souvent sous la plume de Victor Hugo dans ses écrits et ses notes de voyage pour qualifier un paysage ou une ascension : «c'est âpre». Ainsi par exemple dans le carnet employé en 1859 lors du voyage sur l'île de Serk :

Nous débarquons à deux heures quarante minutes au havre Gosselin. [...] À droite et à gauche, deux murailles perpendiculaires, pour fond un plan légèrement incliné. Sur ce plan du fond serpente un sentier encombré de ronces et de pierres tranchantes et roulantes. C'est par là que nous montons. C'est âpre10.

Une âpreté qui va bien au-delà de la rudesse (roughness) revendiquée par William Gilpin dans sa définition du pittoresque, irrégularité qui introduit dans la représentation une diversité et une variété attrayantes ${ }^{11}$. L'âpreté chez Victor Hugo est l'effort manifeste d'un engagement; c'est également l'attention aux aspérités du monde et à la résistance du sensible - «la réalité rugueuse à étreindre», écrira Rimbaud.

Le mouvement impose aussi une précarité. Ici, Hugo sacrifie aux topoï du traditionnel récit de voyage. Dans la lettre à Charles, déjà citée, des bords du Rhin en septembre 1840, on le voit pester contre les matériaux de fortune qu'il est contraint d'utiliser : «Je ne sais pas trop dans quel état arriveront tous les dessins que je vous envoie. Les encres

7. Charles Baudelaire, art. cité, loc. cit, p. 129.

8. Lettre de Victor Hugo à Charles Hugo, 1er octobre 1840; CFL, t. VI, p. 1191. Le dessin est reproduit au tome XVIII, $n^{\circ} 942$.

9. «la souris -167 bre $5 \mathrm{~h} 1 / 4 \mathrm{du}$ s. » pour le folio $40 \mathrm{v}^{\circ}$; « même lieu. $4 \mathrm{~h}$. du soir » pour le folio $41 \mathrm{v}^{\circ}$.

10. Carnet Naf $13450, f^{\circ} 32,1859$; CFL, t. X, p. 1529. Sur ce carnet, voir Jean-Bertrand Barrère, «Un printemps dans l'île de Serk», Victor Hugo à l'œuvre, Paris, Klincksieck, 1965.

11. William Gilpin, Three essays : on picturesque beauty; on picturesque travel; and on sketching landscape : to which is added a poem, on landscape painting, [1792], 1799 pour la traduction française. 


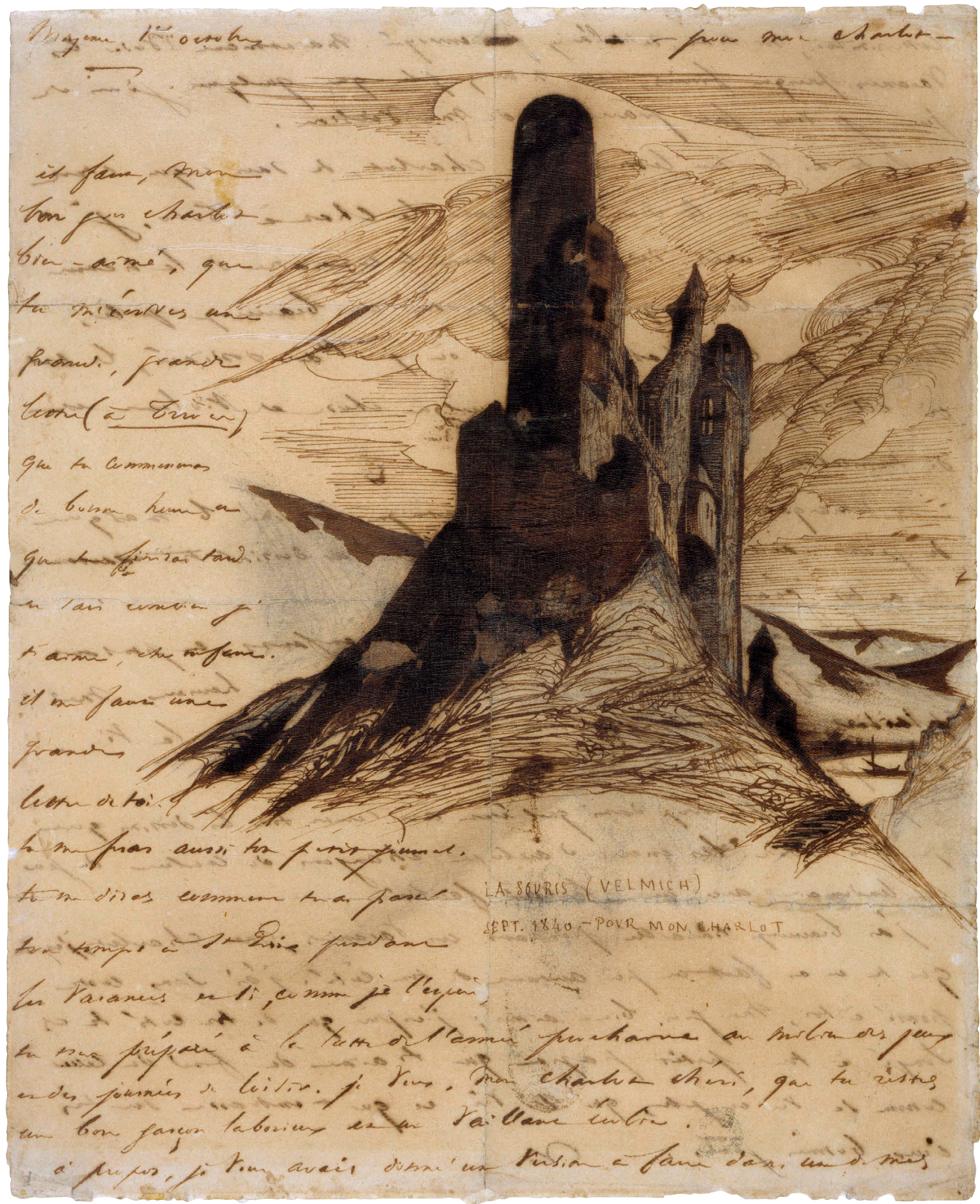

Fig. 1 : Lettre adressée par Victor Hugo à son fils Charles (recto), septembre 1840 ;

«La Souris (Velmich)», plume et lavis d'encre brune, crayon de graphite, papier vélin à filigrane (Paris, Maison de Victor Hugo, MVHP D 0433 ) (C) Maisons de Victor Hugo/Roger-Viollet 


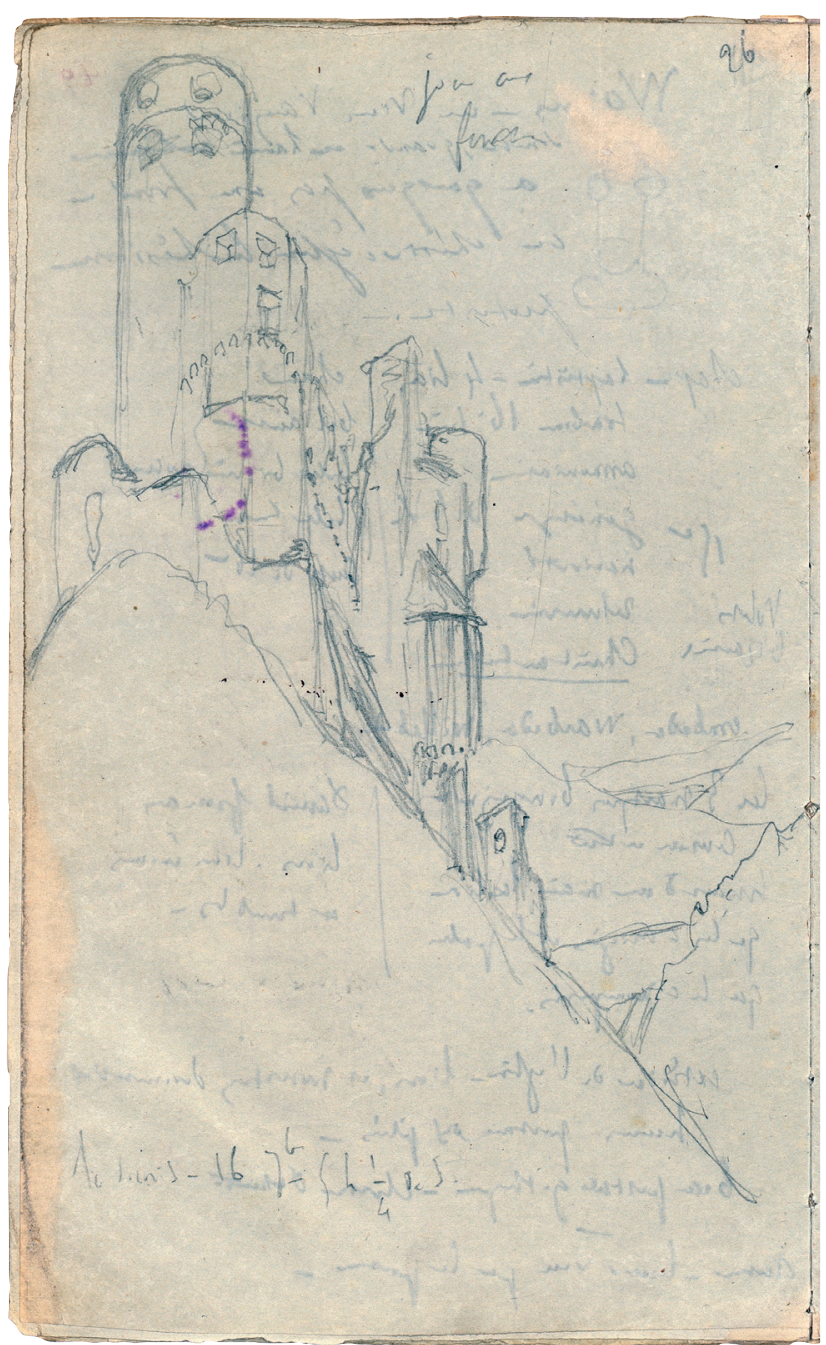

Fig. 2 : [La Souris], 16 septembre $1840\left(\mathrm{BnF}\right.$, Naf 13444, $\left.\mathrm{f}^{\circ} 40 \mathrm{v}^{\circ}\right)$

d'auberge changent de couleur du jour au lendemain avec une fâcheuse facilité12.»

Et Le Rhin conservera sur le mode plaisant la trace littéraire et parodique de cet inconfort d'écriture : «écrire avec une plume qui crache sur du papier qui boit13.» Trait d'époque, assurément. Que l'on pense, par exemple, aux «notes » que Lamartine consent à livrer au lecteur dans son Voyage en Orient. L'écrivain voyageur y pose dans toute la précarité de sa condition, évoquant «le coup d'œil d'un passager assis sur son chameau ou sur le pont de son navire, qui voit fuir des paysages devant lui, et qui, pour s'en souvenir le lendemain, jette quelques coups de crayon sans couleur sur les pages de son journal14 ${ }$.

Mais ce qui se fige en topos littéraire chez Lamartine (l'album de voyage y est «déchiré par les chacals » ou «trempé par l'écume de la mer») et les voyageurs romantiques, demeure vivace et toujours ancré dans l'expérience chez Hugo. Lamartine affectait de considérer que son travail pouvait n'avoir quelque intérêt «que pour des peintres». «Ces notes sont presque exclusivement pittoresques», consignait-il. «C'est le regard écrit». Pour Hugo, cette affirmation ne relève pas de la clause de style. Il se conforme aux pratiques des artistes de son temps : Delacroix, dans ses carnets de voyage en Espagne et en Afrique du Nord, combine notations textuelles et graphiques, le texte apposé à l'emplacement adéquat suppléant l'absence de couleurs ou la maladresse d'une forme hâtivement exécutée 15. Les «carnets nomades 16 » de Victor Hugo portent la trace des contraintes du déplacement dans l'espace, notamment dans le choix de la technique graphique : le crayon ou la mine de plomb sont privilégiés au détriment de la plume ou du lavis - de l'aquarelle dans le cas de Delacroix, techniques réservées aux haltes du soir ou aux stations prolongées.

Les croquis réalisés par Hugo lors d'une excursion à Gavarnie en 1843 illustrent ces pratiques. On sait à quel grandissement épique l'évocation de cette curiosité naturelle sera portée, le cirque pyrénéen se trouvant comparé à l'œuvre du grand «maçon d'apocalypses », Piranèse 17.

12. Voir ci-dessus note 8 et figure 1.

13. Le Rhin, XXXIII ; Victor Hugo, Euvres complètes, éd. Jacques Seebacher et Guy Rosa, Voyages, Paris, Laffont, coll. «Bouquins », 1987, rééd. 2002 [désormais Laffont], p. 335.

14. Alphonse de Lamartine, Souvenirs, impressions, pensées et paysages pendant un voyage en Orient, Ch. Gosselin, 1835, t. I, p. vi.

15. Par exemple, Eugène Delacroix, Album de voyage (Espagne, Maroc, Algérie, janvier-juin 1832), Chantilly, Musée Condé, ms. 390, Base Joconde.

16. Selon la belle expression de Pierre-Marc de Biasi. Voir par exemple «Les carnets de travail de Flaubert : taxinomie d'un outillage littéraire », Littérature, $\mathrm{n}^{\circ} 80,1990$, p. 42-55, et pour une réflexion générale sur le manuscrit hugolien, «Le manuscrit spectaculaire. Réflexions sur l'esthétique hugolienne de la mise en page autographe», Hugo de l'écrit au livre, dir. Béatrice Didier et Jacques Neefs, Saint-Denis, Presses universitaires de Vincennes, coll. «Manuscrits Modernes», 1987, p. 199-217.

17. Dieu; Poésie IV, Laffont, p. 615. 


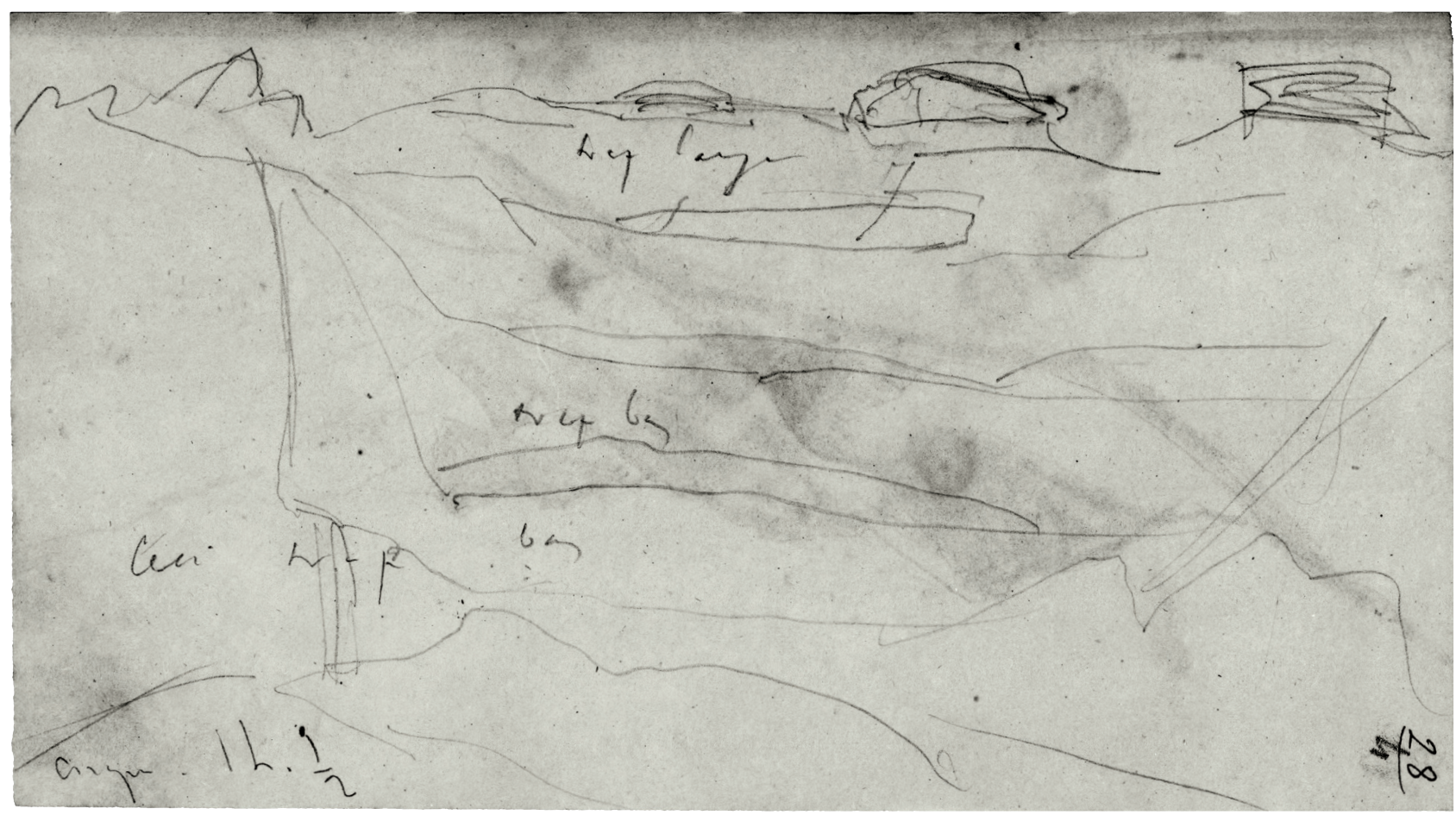

Fig. 3 : [Gavarnie], 1843 (BnF, Naf 13445, f $\left.{ }^{\circ} 28\right)$

Mais le point de départ de ces variations cyclopéennes est à chercher dans l'expérience du voyage elle-même et dans les modestes relevés réalisés par l'écrivain chemin faisant. Le folio 28 du manuscrit Naf 13445 utilisé à l'été 1843 (fig. 3) comporte un dessin sans doute exécuté devant le site : « ceci trop bas », «trop large», «trop bas », lit-on comme commentaires de régie directement apposés sur la feuille 18 .

La séquence qui préside à l'évocation de Gavarnie ${ }^{19}$, entre texte et image, permet d'apprécier la charge cinétique dont sont porteuses les notations graphiques. L'instabilité du trait, les mécanismes d'autocorrection sont symptomatiques d'un observateur en mouvement. En témoigne la remarque du folio $33 v^{\circ}$, consignée non loin de Gavarnie : «après Gèdre en revenant - ligne prise à cheval », qui signale l'importance prise par les conditions de réalisation du croquis tout autant que par la représentation elle-même.

Ces notations ne sont pas rarissimes. Dans l'album Naf $13347, \mathrm{f}^{\circ} 11 \mathrm{v}^{\circ}$, Hugo consigne les péripéties de son périple dans la vallée du Rhône, le 15 octobre 1839. Il n'est pas anodin qu'il adopte, dans les notes qu'il prend, et comme dans l'exemple précédemment cité, un point de vue mobile. Celui, cette fois-ci, d'un passager à bord d'un bateau en proie aux crues du fleuve, du côté de la Voulte (fig. 4). Au verso du feuillet il exécute un rapide croquis du château qui surplombe la localité en multipliant les notations non plus de proportion, mais de tonalité : «sombre», «clair» «gris », ainsi que cette appréciation d'ensemble qui révèle et souligne les effets de contraste entre le château et la vallée du Rhône qui se dessine en arrière-plan : «ciel sombre - bandes lumineuses sur l'horizon 20 ».

18. Datée 31 août 1843 ; CFL, t. XVIII, nº 204.

19. Pour le texte, voir Pyrénées; Voyages, Laffont, p. 856-862.

20. Pour ce texte du voyage de 1839, voir Voyages, Laffont, p. 721-722, et pour le dessin (fig. 4), CFL, t. XVIII, $n^{\circ} 118$. La notice donne «landes» mais il semble plus logique de lire «bandes». 


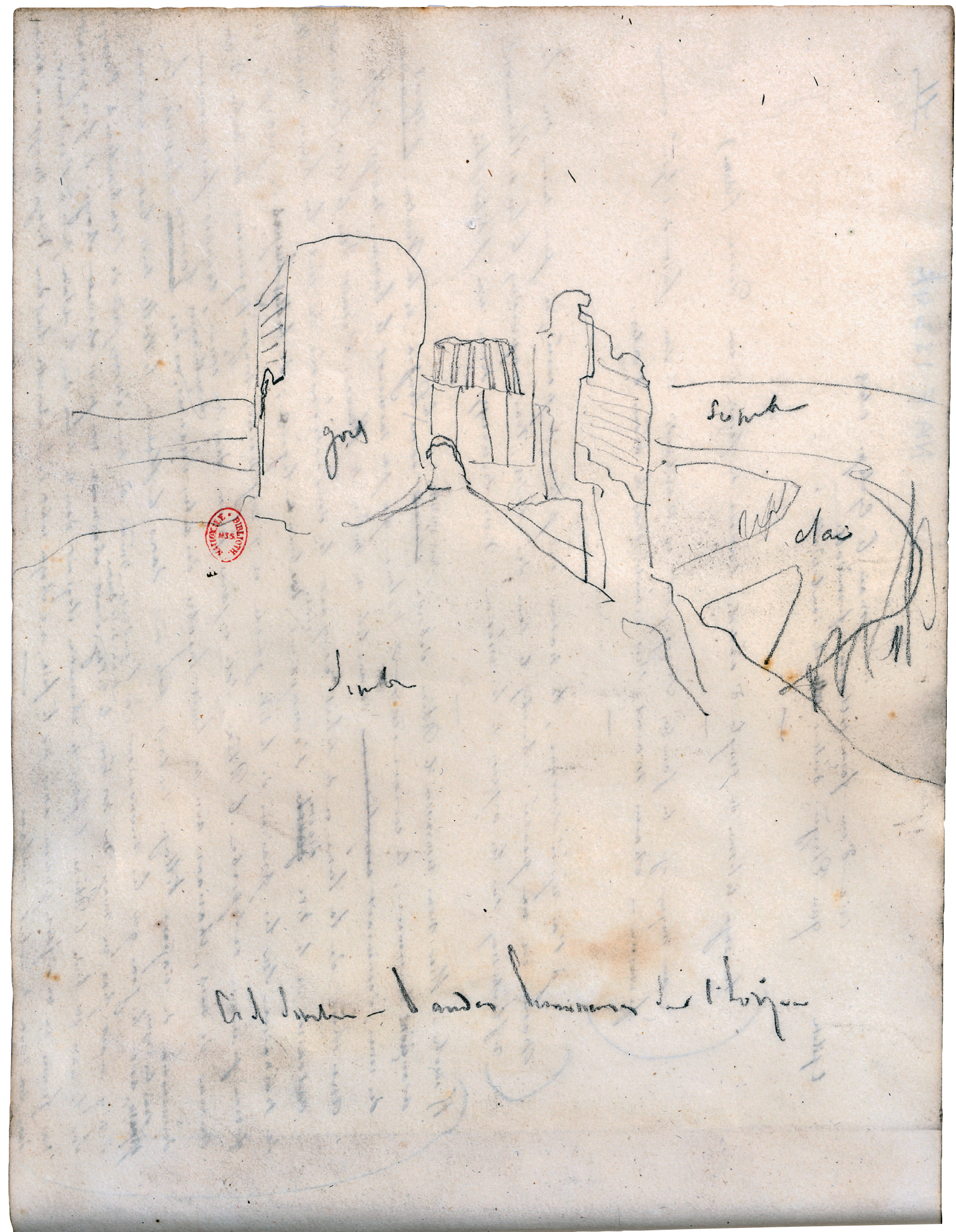

Fig. 4 : [Château de la Voulte], 1839 (BnF, Naf 13347, fo $\left.11 \mathrm{v}^{\circ}\right)$ 
Ce processus d'autocorrection, lorsque le temps manque pour refaire posément un dessin ou même pour corriger les grandes lignes d'une composition mal engagée, permet de noter les rapports, les formes. Le texte supplée alors à l'image, offrant un continuum mixte d'écriture et de dessin.

Les croquis hugoliens des carnets de voyage ou de travail conservent donc la trace d'un point de vue d'observateur, d'une posture de voyageur. Ils absorbent en quelque sorte une charge cinétique qui se trouve parfois restituée à distance dans l'œuvre littéraire. Ainsi, la première phrase du livre des Misérables consacré à Waterloo n'est-elle pas qu'un biais commode pour présenter au lecteur la topographie du champ de bataille :

L'an dernier (1861), par une belle matinée de mai, un passant, celui qui raconte cette histoire, arrivait de Nivelles et se dirigeait vers La Hulpe. Il allait à pied. Il suivait, entre deux rangées d'arbres, une large chaussée pavée ondulant sur des collines qui viennent l'une après l'autre, soulèvent la route et la laissent retomber, et font là comme des vagues énormes. Il avait dépassé Lillois et Bois-Seigneur-Isaac. Il apercevait, à l'ouest, le clocher d'ardoise de Braine-l'Alleud qui a la forme d'un vase renversé. Il venait de laisser derrière lui un bois sur une hauteur, et, à l'angle d'un chemin de traverse, à côté d'une espèce de potence vermoulue portant l'inscription : Ancienne barrière $n^{\circ} 4$, un cabaret ayant sur sa façade cet écriteau : Au quatre vents. Échabeau, café de particulier ${ }^{21}$.

Ce préambule correspond à une expérience vécue que l'écriture romanesque restitue, mais qui a en amont transité par la marche et le dessin. Le travail d'écriture du roman, interrompu en février 1848, n'avait pas encore donné l'occasion d'une évocation de la bataille de Waterloo. Hugo décrivait, dans le chapitre «Un des spectres rouges de ce temps-là », les états de service de Pontmercy. Mais rien ne laissait augurer ce que serait le chantier Waterloo, durant l'exil. C'est semble-t-il au moment de la relecture du manuscrit, en vue de sa reprise et de sa poursuite que naît l'idée d'un «grand récit épique mêlé au roman 22 ». Au travail de documentation livresque auquel se livre alors Hugo s'adjoint la nécessité d'une visite sur place. L'écrivain se rend à Mont-Saint-Jean le 7 mai 1861. De là, il part visiter le champ de bataille de Waterloo, sur lequel il retournera le 16. Il écrit le 20 à son fils François-Victor : «Je suis ici

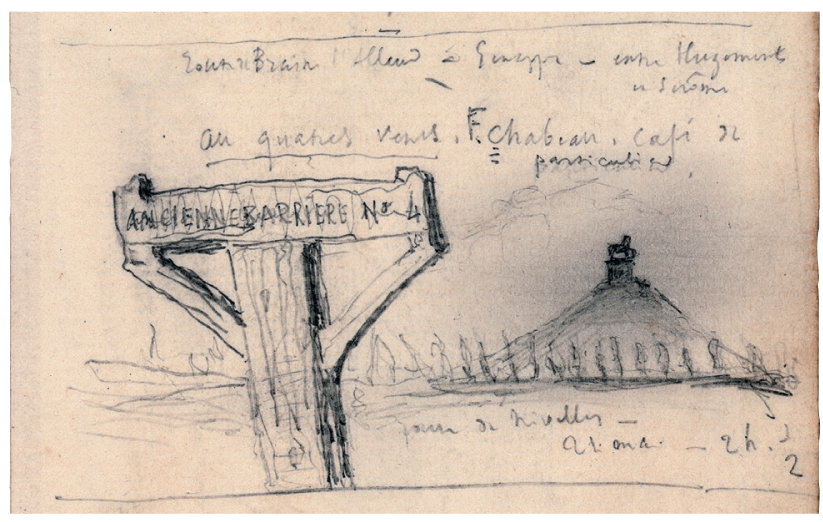

Fig. 5 : «Ruine de Nivelles. 21 mai. 2 h $1 / 2 »(B n F$, Naf 13452, fo 52)

près de Waterloo. Je n'aurai qu'un mot à en dire dans mon livre, mais je veux que ce mot soit juste. Je suis donc venu étudier cette aventure sur le terrain, et confronter la légende avec la réalité23.»

Le carnet utilisé d'avril à juillet $1861^{24}$ lors du séjour de Hugo à Mont-Saint-Jean comporte des notes et croquis essentiels pour la rédaction du livre I de la Deuxième Partie, «Waterloo», entreprise du 8 au 21 décembre 186125. L'acuité du point de vue, telle qu'elle se manifeste

21. Les Misérables, II, I, 1 ; Roman II, Laffont, p. 241.

22. Ms. Naf 24744, f 643, BnF; Chantiers, Laffont, p. 736. Pour les détails de cet épisode, voir Delphine Gleizes, «Images et construction du romanesque chez Victor Hugo : "Ce qu'enseignent les enseignes" », Victor Hugo et le romanesque, sous la direction d'Agnès Spiquel, Paris-Caen, Minard, Lettres Modernes, Études romanesques 9, 2005, p. 31-45.

23. Victor Hugo à François-Victor Hugo, 20 mai 1861; CFL, t. XII, p. 1117.

24. Ms. Naf 13452, BnF. Pour le texte, voir Chantiers, Laffont, p. 882-902, et pour l'illustration (fig. 5), CFL, t. XVIII, n 242.

25. Sur la rédaction de l'épisode, voir Jean-Marc Hovasse, «Waterloo et Victor Hugo : genèse poétique », «La chose de Waterloo », Une bataille en littérature, dir. Damien Zanone, Leiden/Boston, Brill, Rodopi, 2017, p. 87-110. Pour la conception des Misérables, voir René Journet et Guy Robert, Le Manuscrit des «Misérables», Annales littéraires de l'Université de Besançon, $\mathrm{n}^{\circ}$ 61, 1963 ; le dossier des Misérables par René Journet (Chantiers, Laffont, p. 727-967); Guy Rosa, «Outils de travail : les carnets de travail des Misérables », Carnets d'écrivains I, dir. Louis Hay, Pierre-Marc de Biasi et al., Paris, Éditions du CNRS, 1990, p. 99-118; Jean Gaudon, «Croquis, dessins, griffonnages. Les notations graphiques de Victor Hugo », De la lettre au livre, Sémiotiques des manuscrits littéraires, dir. Louis Hay, Paris, CNRS, 1989, p. 115-139. 
dans le texte final des Misérables, tient à la façon dont les dessins ont été réalisés par le marcheur de MontSaint-Jean. Ainsi par exemple du petit croquis du folio 52 (fig. 5) daté du «21 mai. 2 h 1/2» et localisé à la «Ruine de Nivelles». Le dessin comporte un traitement des plans particulièrement intéressant. Au premier, une pancarte portant l'inscription «ANCIENNE BARRIERE $N^{\circ} 4$ ». En plan intermédiaire, un rideau d'arbres qui vient hachurer la plaine, tandis que se dessine en arrière-plan, légèrement ombrée par la mine de plomb, la silhouette du lion de Waterloo. La dynamique du point de vue est donc marquée par cette succession d'écrans que le regard doit traverser ainsi que par la notation manuscrite qui accompagne le dessin : «Route de Braine l'Alleud à Genappe - entre Hugomont et Jérôme. Au quatres vents, F. Chabeau. Café de particulier.»

C'est ce mouvement consigné et comme recélé par la configuration spatiale du carnet qui se trouve restitué dans le roman, à la fin du premier chapitre, le cheminement de l'observateur allant de pair avec l'effort pour discerner les plans du paysage :

Le passant se redressa. Il fit quelques pas et s'en alla regarder au-dessus des haies. Il aperçut à l'horizon à travers les arbres une espèce de monticule et sur ce monticule quelque chose qui, de loin, ressemblait à un lion.

Il était dans le champ de bataille de Waterloo 26.

Autre exemple de restitution dans l'univers romanesque d'une charge cinétique saisie par le dessin. Ici, le cas est d'autant plus notable que cette prise en compte du mouvement de l'observateur ne passe plus comme dans le cas de Waterloo par un mécanisme de représentation mais par un processus de symbolisation. Il s'agit de plusieurs feuillets des manuscrits Naf 13460 et 13459 , ayant servi, en 1864, à la conception des Travailleurs de la mer. À plusieurs reprises, Hugo dessine dans ses carnets l'île de Guernesey ainsi que les îlots qui forment l'archipel 27 . Le carnet Naf 13460 , commencé en décembre 1863 et achevé dans les premiers mois de 1864, est en partie consacré à William Shakespeare, mais dans les dernières pages, repassées à l'encre sur une première couche de crayon à papier, se trouvent deux cartes.

Sur la première, au folio $82 \mathrm{v}^{\circ}$ (fig. 6), est représentée la côte ouest de Guernesey, de Saint-Sampson au nord à

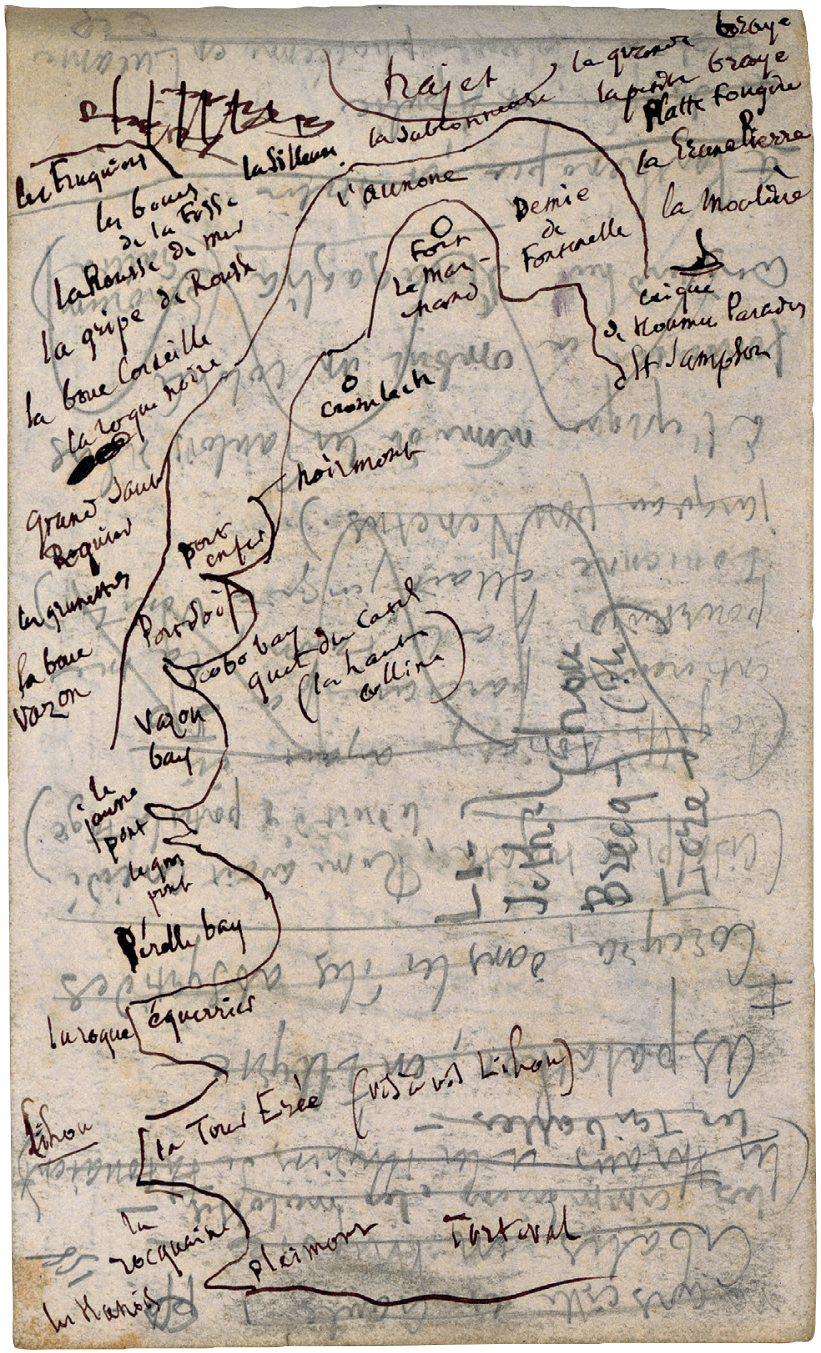

Fig. 6 : [Carte partielle de Guernesey] (BnF, Naf 13460, $\left.\mathrm{f}^{\circ} 82 \mathrm{v}^{\circ}\right)$

Torteval au sud en passant par Plainmont. Hugo y énumère avec minutie tous les noms de baies et de caps qui rythment le rivage; il mentionne également les écueils et rochers qui se situent en face de chaque cap. Le mot «trajet» est mentionné en haut du feuillet et se trouve associé à un trait

26. Les Misérables, II, I, 1 ; Roman II, Laffont, p. 242.

27. Ces croquis de 1'archipel anglo-normand avaient été précédés d'une carte, réalisée lors du voyage sur l'île de Serk en 1859 (Naf 13 450, f 84). 


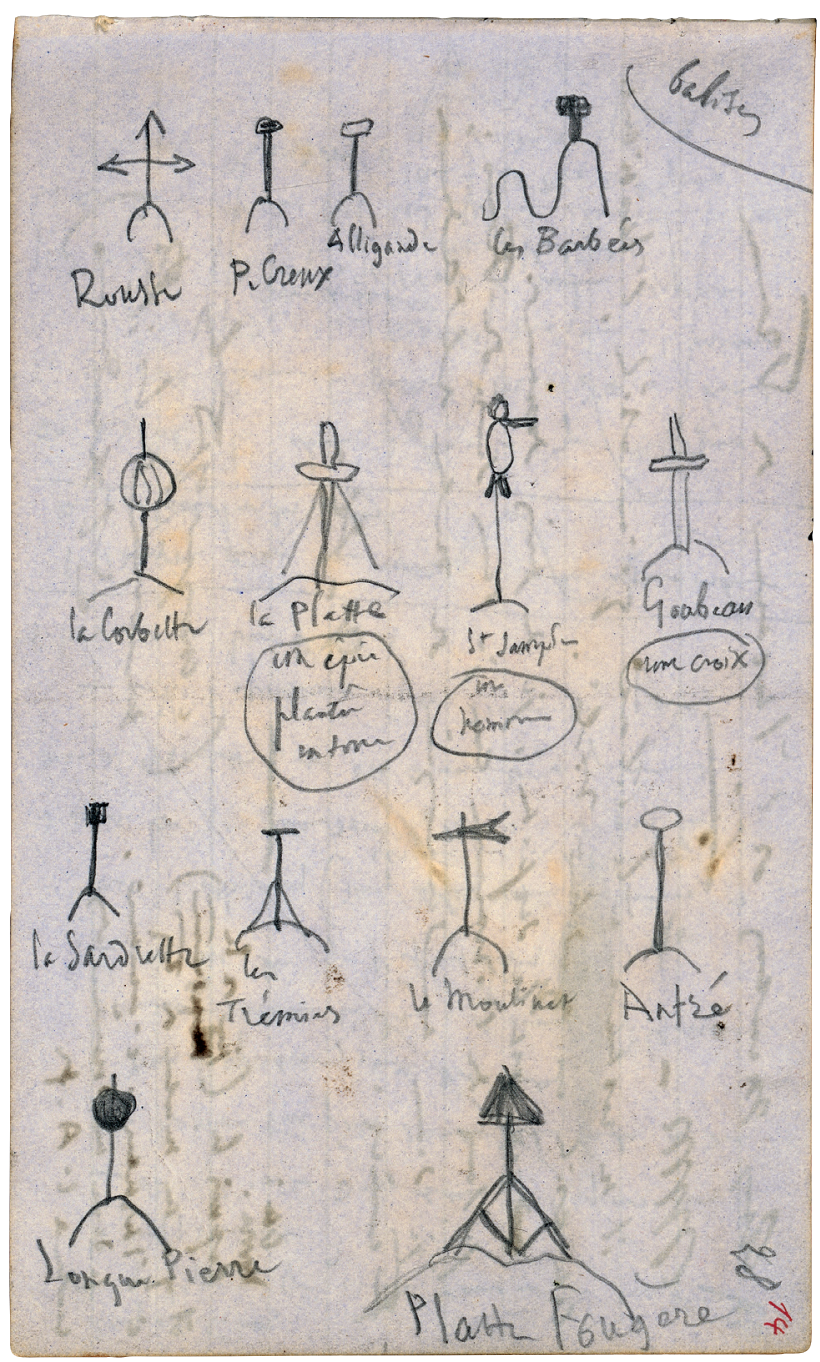

Fig. 7 : Balises (BnF, Naf 13459, $\left.\mathrm{f}^{\circ} 14\right)$

qui part de Saint-Sampson et qui suit partiellement la côte ouest pour s'interrompre ensuite au niveau de Vason Bay. Il est plus que probable que ce trait matérialise le parcours dans le roman du marin Gilliatt pour rejoindre l'écueil des Douvres sans être aperçu depuis Saint-Pierre-Port28. Cette appréhension cartographique et symbolique de l'île se retrouve sur le carnet Naf 13459 , directement consacré à la rédaction du roman à partir du 24 mai 1864. On y découvre trois cartes au crayon ${ }^{29}$ qui proposent à nouveau un relevé

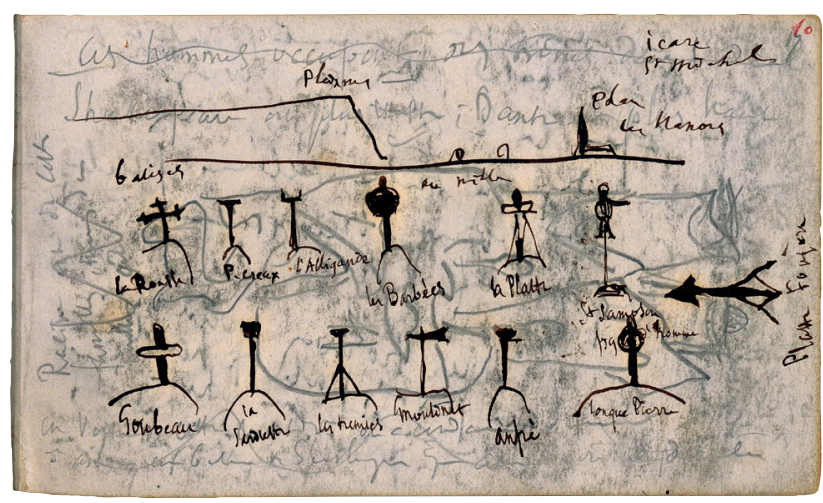

Fig. 8 : [Balises] (BnF, Naf 13460, fo 10$)$

des côtes de l'archipel. À ces indications topographiques sont également associées les listes des balises qui jalonnent la côte en regard des écueils : $\mathrm{f}^{\circ} 10$ du manuscrit Naf 13460 et $\mathrm{f}^{\circ} 14$ du manuscrit Naf 13459 (fig. 7 et 8).

Tout comme dans le cas précédemment évoqué des Misérables, ces relevés sont une manière de consigner une perception spatiale en lien avec les déplacements de l'écrivain : ce qui est emmagasiné par l'expérience du promeneur et de l'observateur dans les cartes et listes est en attente d'une exploitation romanesque. L'inventaire est une façon de ressaisir abstraitement le trajet tout à la fois effectif et mental que l'écrivain a pu réaliser. Il n'exprime pas à proprement parler le mouvement mais il le «stocke» : la mention des diverses balises se trouvera éparpillée au fil des chapitres dans le texte définitif et servira de point de repère pour évoquer les déambulations du héros Gilliatt ${ }^{30}$. Le mouvement est donc «incorporé» au dessin et symétriquement le dessin «prend corps », restitue la présence physique qui lui donne naissance.

28. Voir Les Travailleurs de la mer, I, VIII, 2, «Beaucoup d'étonnement sur la côte ouest ».

29. Naf $13459, \mathrm{f}^{\circ} 9 \mathrm{v}^{\circ}, 10 \mathrm{v}^{\circ}$ et $11 \mathrm{v}^{\circ}$. Des précisions sont données par Yves Gohin dans son édition des Travailleurs de la mer (Paris, Gallimard, coll. «Bibliothèque de la Pléiade», 1975, p. 1502, n. 2).

30. Voir Les Travailleurs de la mer, I, I, 6, et I, VII, 2 ; Roman III, Laffont, p. 64 et $182-184$. 
Présence d'un corps, tout à la fois intempestive et structurante, que la ligne - qu'elle soit de dessin ou d'écriture - enregistre à la manière d'un tracé de sismographe. Hugo a pu se plaire à théâtraliser la corporéité de son écriture, le jeu nerveux de la main qui trace. Dans Le Rhin encore, il évoque «l'imagination ailée, opulente et joyeuse d'un homme à pied», «Musa pedestris ${ }^{31}$ », et en guise d'illustration relate une mésaventure ancienne qui lui serait arrivée du côté de Claye, dans les années 1830. L'écrivain se dépeint interrompant sa marche pour s'asseoir, «à un endroit où le chemin tourne brusquement», «adossé à un chêne, sur un talus d'herbe, les pieds pendant dans un fossé» afin de «crayonner» sur son livre vert. À la manière d'un généticien en campagne, il fait mine d'examiner a posteriori les lignes anodines qu'il traçait alors et y devine une autre histoire, matérielle, que celle qu'il entreprenait d'écrire. C'est que bientôt un ours, puis deux, puis trois, étaient venus troubler son travail, suscitant surprise et inquiétude :

Je confesse qu'à cette nouvelle apparition, qui élevait mes perplexités à la seconde puissance, ma main trembla. J'étais en train d'écrire cette ligne : «... peuvent entendre passer les sérénades. » Sur mon manuscrit je vois aujourd'hui un assez grand intervalle entre ces mots : «entendre passer» et ces mots : «les sérénades. » Cet intervalle signifie - Un deuxième ours 32 !

Ce cas n'est pas isolé. Sur la page, pour paraphraser l'un des chapitres des Travailleurs de la mer, «l'inattendu intervient». C'est le corps de l'écrivain dessinateur qui se manifeste. «Je pique ma canne dans les landes et j'écris debout ${ }^{33}$ », note Hugo lors de ses excursions au Pays basque. L'inattendu qui guide la main du dessinateur, c'est ce lapin (fig. 9) se présentant au poète en excursion tandis qu'il compose quelques vers et dont il réalise l'esquisse au verso de la page ${ }^{34}$.

C'est encore le corps du poète - ici non pas du marcheur, il est vrai, mais de l'écrivain à sa table de travail - que semble mettre en scène le manuscrit du poème des Voix intérieures, «À des oiseaux envolés», en avril 1837. Hugo raconte comment il chasse ses enfants, mécontent qu'ils aient fait brûler dans l'âtre, par jeu, quelques-uns de ses vers égarés sur un copeau de papier. Le poète se repent bien vite de ce moment d'agacement, et implore les garnements de revenir :

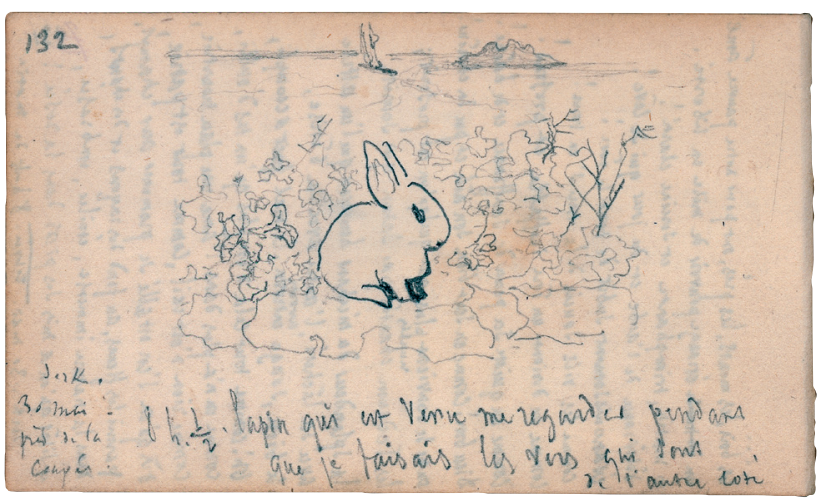

Fig. 9 : [Lapin] (BnF, Naf 13450, $\left.\mathrm{f}^{\circ} 70 \mathrm{v}^{\circ}\right)$

Espiègles radieux que $\mathrm{j}$ 'ai fait envoler,

Oh! revenez ici chanter, danser, parler,

Tantôt, groupe folâtre, ouvrir un gros volume,

Tantôt courir, pousser mon bras qui tient ma plume,

Et faire dans le vers que je viens retoucher

Saillir soudain un angle aigu comme un clocher

Qui perce tout à coup un horizon de plaines 35 .

L'écriture enregistre à son corps défendant la «secousse du réel ${ }^{36}$ », le coup de coude de la marmaille indisciplinée. Une irruption intempestive qui trouve à se formuler également en termes graphiques dans le manuscrit : les vers laissent la place à un dessin dans lequel l' «angle aigu comme un clocher» vient saillir dans un «horizon de

31. Le Rhin, XX ; Voyages, Laffont, p. 136-137.

32. Ibid., p. 137

33. Pyrénées; ibid., p. 810.

34. Légendes : «Serk. / 30 mai [1859] -/ près de la / Coupée. » Et : « 8 h. 1/2. lapin qui est venu me regarder pendant / que je faisais les vers qui sont / de l'autre côté» (CFL, t. XVII, n² 236).

35. «À des oiseaux envolés», Les Voix intérieures, XXII ; Poésie I, Laffont, p. 877. Pour le dessin (fig. 10), CFL, t. XVIII, $n^{\circ} 488$.

36. L'expression se trouve dans Promontorium somnii, et traduit la façon dont le rappel à la réalité constitue un contrepoint - un contrepoids salutaire à la perte de repères induite par la contemplation (Critique, Laffont, p. 640). Voir à ce propos Yves Gohin, Victor Hugo, Paris, PUF, coll. «Que sais-je ?», 1987. Nous avions également abordé cette question, avec Pierre Georgel, à l'occasion de la communication «Pictura et poesis chez Victor Hugo : au-delà du paragone» au colloque Vers en images, organisé par Serge Linarès, Robert Kahn et Christophe Martin du 14 au 16 octobre 2010 à l'université de Rouen. 


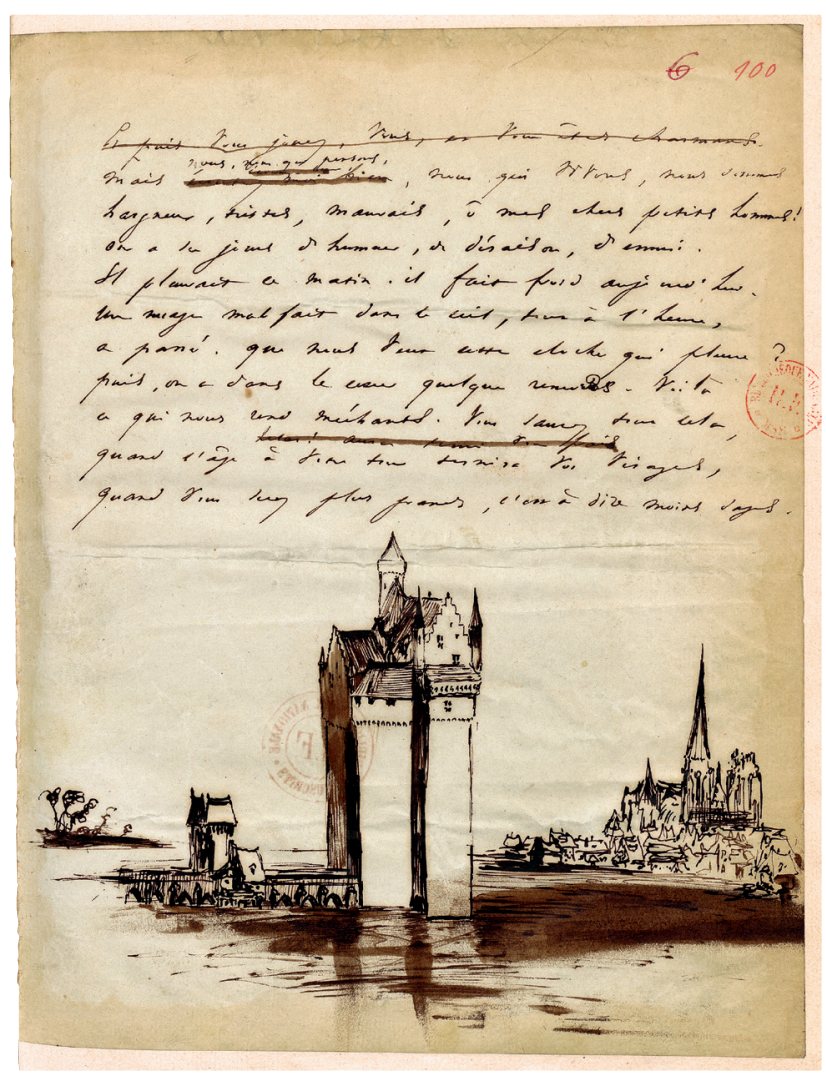

Fig. 10 : «À des oiseaux envolés» (manuscrit des Voix intérieures, $\left.\mathrm{BnF}, \mathrm{Naf} 13361, \mathrm{f}^{\circ} 100\right)$

plaines» (fig. 10). Sur le feuillet même où s'écrit «À des oiseaux envolés », et sans qu'il soit possible d'en déterminer génétiquement l'antériorité ou la postériorité, un paysage dessiné, analogue à celui évoqué par le poème, s'ébauche dans l'espace de la page laissé libre par les vers.

Le manuscrit met en scène un corps en mouvement, le carnet enregistre les secousses erratiques du marcheur et transmet les cahots de la route au «chaos dans le pinceau ${ }^{37}$ ». Mais si le dessin peut traduire la présence d'un corps - sa marche inspirante - il figure aussi parfois la façon dont ce même corps s'absente, question avivée encore par l'expérience de l'exil. La préface des Contemplations le rappelait puissamment, c'est l'œuvre d'un «esprit qui marche de lueur en lueur» et «qui s'arrête éperdu "au bord de l'infini" », formule qui évoque tout à la fois le mécanisme de la rêverie contemplative conçue comme abstraction du réel sensible et la condition du proscrit qui le contraint à ne pouvoir fouler qu'en poèmes et dessins le sol de France. La démarche de l'esprit prend le pas sur la marche du corps. Elle en conserve le dynamisme mais en efface la présence, tantôt suivant «la pente de la rêverie», tantôt s'engageant dans un pénible pèlerinage mental. À cette double tentation font écho deux modalités d'expression graphique qui correspondent à une dominante de l'exil, sans en être non plus l'exclusive : le lavis et le pochoir.

Paradoxalement, le phénomène contemplatif est souvent associé à la marche, comme si la régularité du pas avait sur le promeneur le même effet hypnotique que le choc des rames battant la surface de l'eau dans Les Rêveries de Rousseau. À force de rythme et de cadence, le corps s'efface. C'est la marche du pâtre dans «Magnitudo Parvi», lorsque «Contempler les choses, / C'est finir par ne plus les voir». C'est encore le souvenir - une fois de plus matriciel - du voyage aux Pyrénées de 1843, dans lequel le poète relate sa propre expérience :

Je marchais dans la montagne sans trop savoir où j'étais; peu à peu le paysage extérieur que je regardais vaguement avait développé en moi cet autre paysage intérieur que nous nommons la rêverie; j'avais l'œil tourné et ouvert au dedans de moi, et je ne voyais plus la nature, je voyais mon esprit 38 .

Un glissement s'opère sur la «pente insensible», qui va «du monde réel à la sphère invisible ${ }^{39}$ ». Or il existe une connivence profonde entre cette diffusion progressive de la contemplation et la pratique du lavis. La rêverie n'y est pas représentée. C'est l'élaboration même du dessin qui y renvoie et la restitue, en en faisant l'objet non plus d'une mimesis, mais d'une poïesis. Chaque technique présuppose un certain rapport au monde. En un sens, le dessin à la mine est une manière de circonscrire les aspérités du terrain, de rejouer un corps à corps avec l'âpreté du monde; la technique à l'huile

37. L'expression qualifie dans Les Misérables les «puissants peintres » qui sont seuls capables de rendre la vérité d'une bataille - ici Waterloo. 38. Pyrénées; Voyages, Laffont, p. 791.

39. «La Pente de la rêverie», Les Feuilles d'automne, XXIX; Poésie I, Laffont, p. 631. 


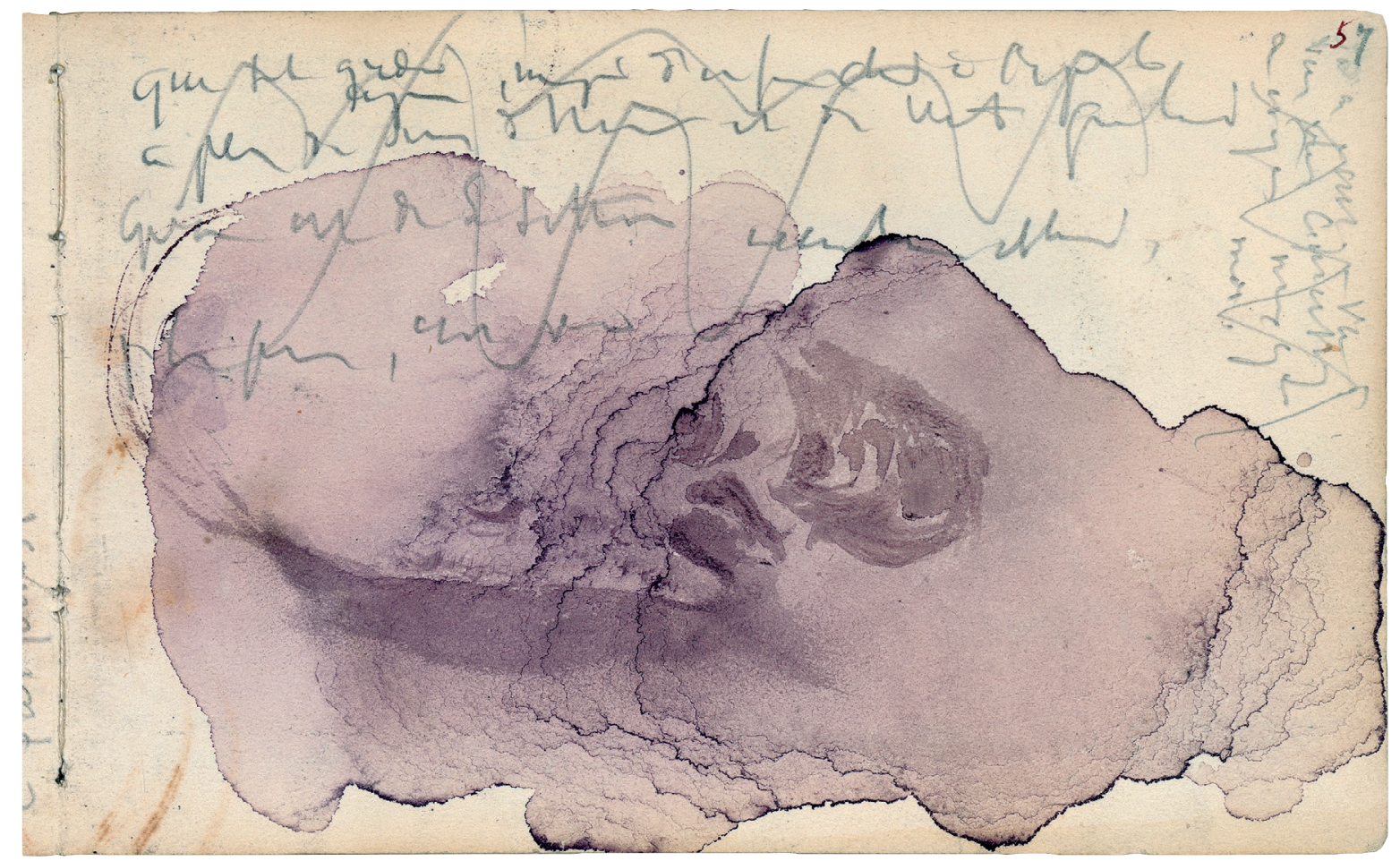

Fig. 11 : [Lavis d'encre violette] (BnF, Naf 13476, fo 5)

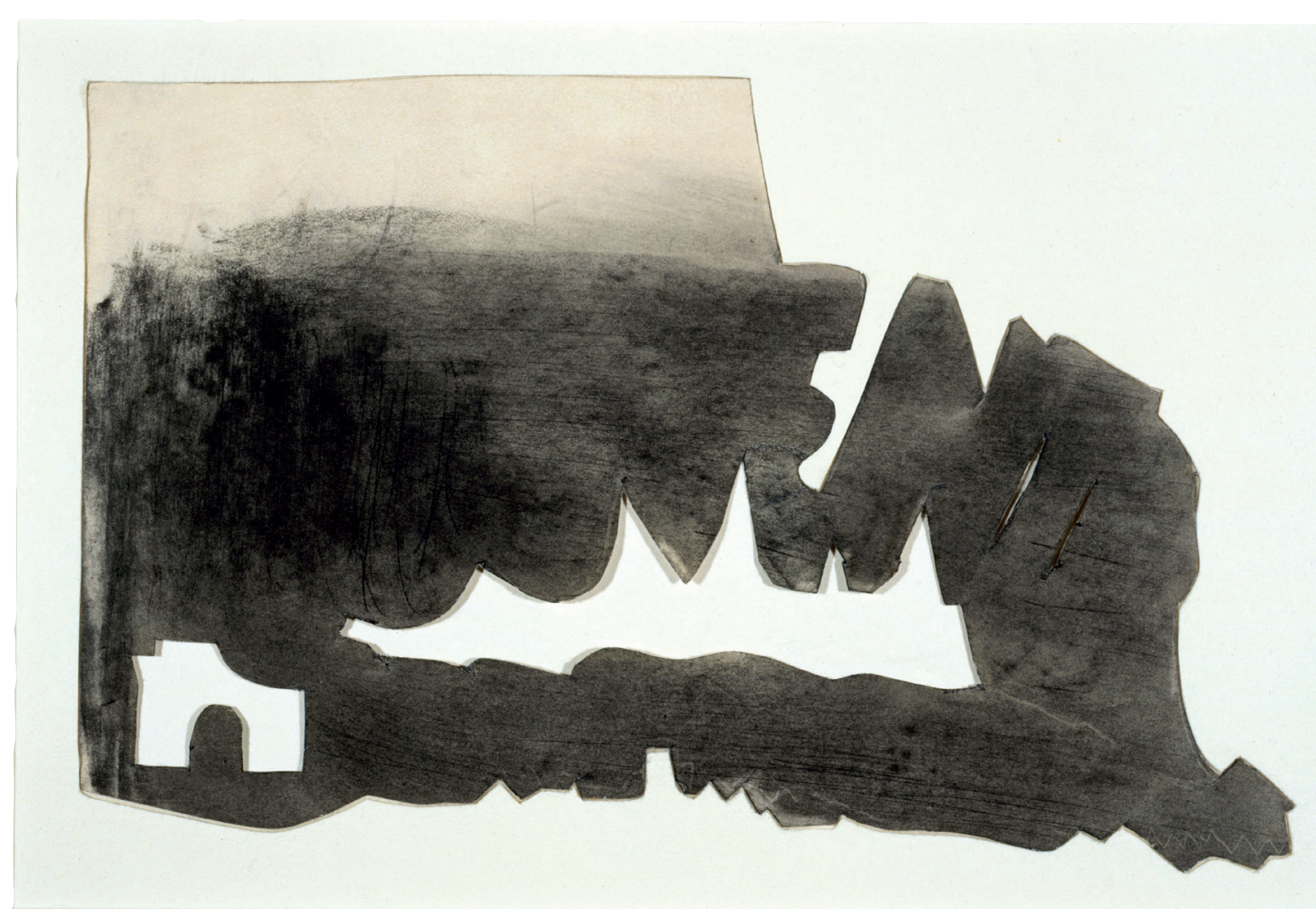

Fig. 12 : «Souvenir», Pochoir, vers 1863-1864 (Paris, Maison de Victor Hugo, MVHP D 2648) (C) Maisons de Victor Hugo/Roger-Viollet 


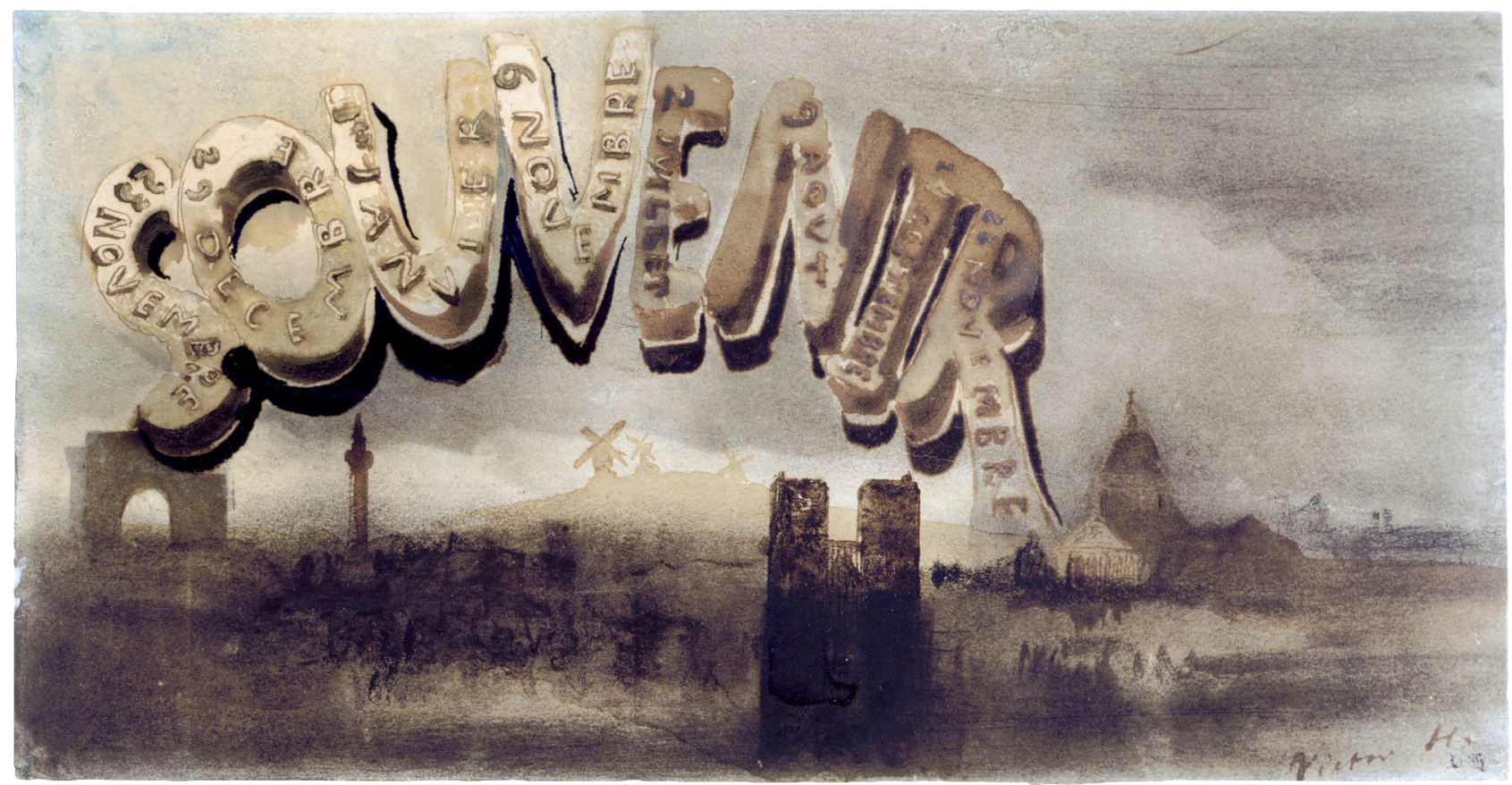

Fig. 13 : «Souvenir», vers 1863-1864 (Maison de Victor Hugo, MVHP D 0926) (C) Maisons de Victor Hugo/Roger-Viollet

conserve la marque du geste - la touche - ou l'escamote dans l'uniformité d'un glacis. Quant au lavis, il y a dans son mode de propagation de l'encre diluée, dans cette capillarité invasive, quelque chose d'une genèse, qui commence au dépôt du liquide sur la feuille, s'étend, s'immobilise enfin, sèche. Le lavis conserve le fantôme de cette progression suspendue (fig. 11) ${ }^{40}$. Se devinent les cernes d'une encre par cercles asséchée. Un processus dont la main a engagé les prémices mais dont elle n'a pas sanctionné l'achèvement, analogue à cette forme de déprise si profondément à l'œuvre dans le mécanisme contemplatif.

L'autre procédé graphique qui traduit - et trahit - des absences est la technique du pochoir, dont l'usage se manifeste dans la seconde moitié des années $1850^{41}$. Il s'agit de découpages entrant dans la réalisation de compositions à l'encre et/ou à la mine de plomb : le pochoir, comme l'a souligné Pierre Georgel, permet le développement d'une esthétique de la silhouette 42 . Il opère également selon une technique de réserve à partir de laquelle le dessin peut ensuite être retravaillé. Ces compositions portent la trace d'un objet manquant et il n'est pas anodin qu'elles soient souvent associées à des processus de remémoration. Ainsi le dessin adressé à Léonie d'Aunet, Souvenir 43 (fig. 12 et 13), et plus encore le dessin intitulé France (fig. 14 et 15), significativement monté sur un exemplaire des Contemplations.
Dans l'un et l'autre cas, les dessins se trouvent associés à un paysage rêvé que l'esprit parcourt dans une sorte de pèlerinage mental. Souvenir offre la vision d'un panorama de Paris et s'ouvre sur des jours doublement révolus : ceux de la liaison avec Léonie d'Aunet et ceux d'une vie dans la capitale d'avant l'exil. «Depuis bien des années déjà, Victor Hugo n'est plus parmi nous », écrivait Baudelaire... La ligne des monuments de Paris qui s'ébauche dans un brumeux sfumato n'est pas sans rappeler, et par la datation et par le contenu, le chapitre des Misérables «Les zigzags de la stratégie» $(\mathrm{I}, \mathrm{V}, 1)$ : «Voilà bien des années déjà que l'auteur de ce livre, forcé à regret de parler de lui, est absent de Paris », note Victor Hugo 44 , qui fait ressurgir, dans les pages qui suivent, le «Paris ancien» «qu'il a religieusement

40. Ce lavis de 1874 est reproduit (en noir et blanc) dans CFL, t. XVIII, $\mathrm{n}^{\circ} 972$.

41. Voir ibid., n 860-879.

42. Pierre Georgel, «La vision en silhouette», L’Arc, ${ }^{\circ}$ 56, 1976 , p. 25-32.

43. Sur la série des dessins «souvenirs», voir Jean Gaudon, «Souvenir de...», Victor Hugo et les images, dir. Madeleine Blondel et Pierre Georgel, Ville de Dijon, Aux Amateurs des Livres, 1989, p. 153-167.

44. Sur les échos entre Baudelaire et Hugo autour de 1860, voir Jean-Marc Hovasse, «Les signes de Hugo au cygne de Baudelaire», dans Choses vues à travers Hugo, hommage à Guy Rosa, dir. Claude Millet, Florence Naugrette et Agnès Spiquel, Presses universitaires de Valenciennes, 2007, p. 367-376. 
emporté dans sa mémoire». Mesure-t-on pour un exilé la force de révolte d'une hypotypose? C'est que Hugo revivifie la pratique des arts de mémoire 45 , déambulant dans l'espace mental que son exil lui interdit d'éprouver dans la réalité. Refaire le trajet, retrouver les étapes d'un cheminement. Le poème des Contemplations «À celle qui est restée en France», rappelle ce passage de l'actuel au virtuel qu'est l'exil, interdisant désormais au poète de se recueillir sur la tombe de sa fille :

Ainsi, ce noir chemin que je faisais, ce marbre

Que je contemplais, pâle, adossé contre un arbre,

Ce tombeau sur lequel mes pieds pouvaient marcher,

La nuit, que je voyais lentement approcher,

Ces ifs, ce crépuscule avec ce cimetière,

Ces sanglots, qui du moins tombaient sur cette pierre,

Ô mon Dieu, tout cela, c'était donc du bonheur 46 !

«Demain, dès l'aube... » retrace également ce processus de virtualisation qu'impose la proscription. Le poème, daté dans l'édition du 3 septembre 1847 qui fait coïncider le temps de l'énonciation - «je partirai» - avec la veille du pèlerinage anniversaire de la mort de Léopoldine, porte en réalité sur le manuscrit la date du 4 octobre 1847 . Il célèbre le voyage effectif que Hugo réalisa pour se rendre à cette date sur la tombe de sa fille à Villequier. Cependant, la publication du poème dans le contexte de l'exil, en 1856, dans Les Contemplations, modifie les données de l'énonciation : ce qui se donne à lire n'est plus le témoignage d'un trajet réel, mais la trace d'un voyage mental. Effectuation d'un pèlerinage en esprit dont Hugo restitue précisément les stations, qui sont autant de manifestations du visible : «l'or du soir qui tombe» sur la Seine ou «les voiles au loin descendant vers Harfleur».

C'est la force tragique du poème qui tient à cet écart entre les circonstances de sa genèse et le dispositif de sa publication : «Je partirai» ou la résistance d'un futur qui se refuse à devenir un conditionnel. À bien des égards, «Demain, dès l'aube...» peut apparaître comme un poème-pochoir : le réel s'est retiré et il ne reste que la trace fantomatique mais obstinée de son absence.

Un mécanisme identique préside à la création, postérieure de quelques années, du dessin «France», réalisé au pochoir et à l'encre (fig. 14 et 15$)^{47}$. La stèle du tombeau y est figurée en réserve, épargnée par le cache du pochoir

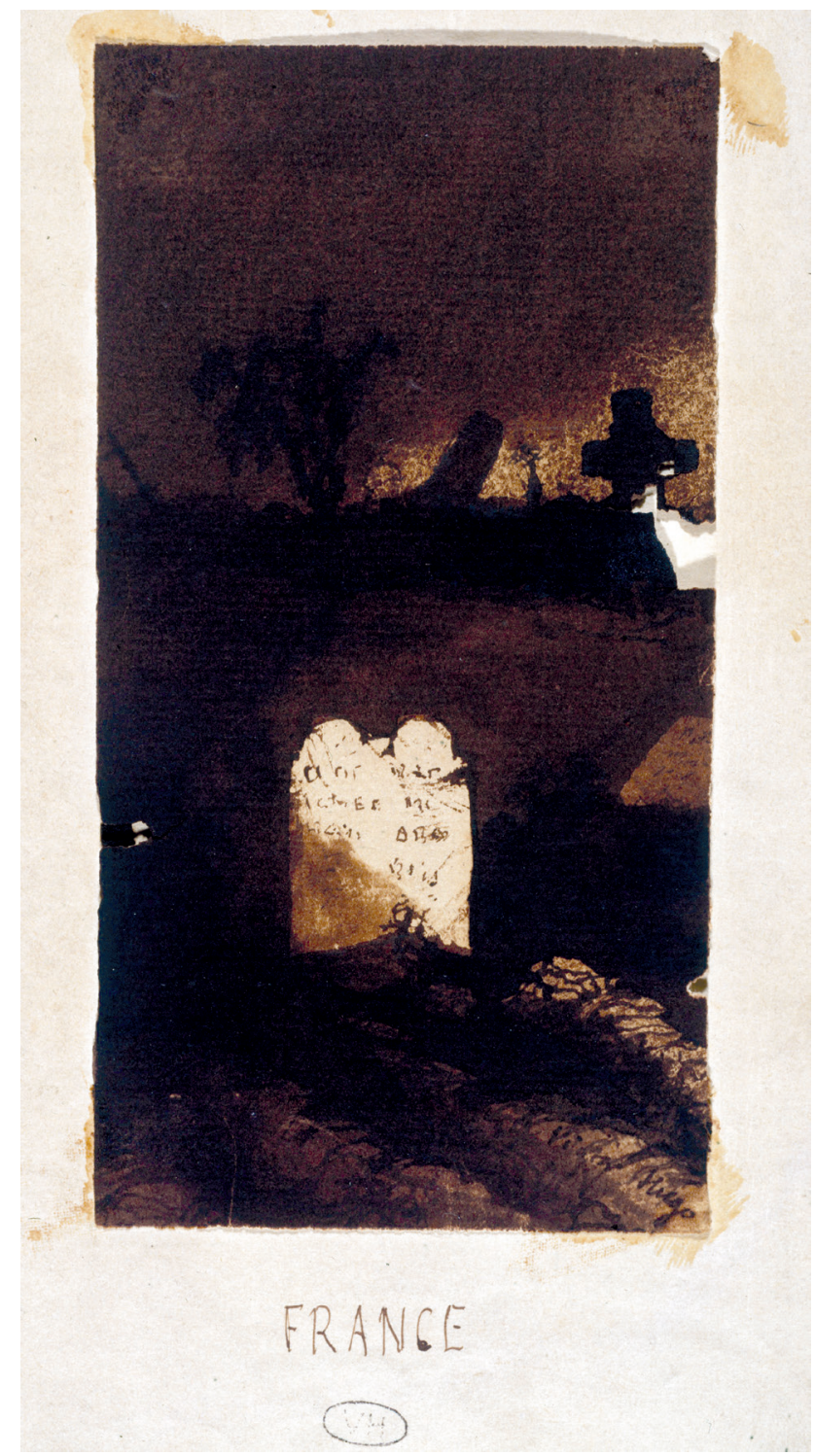

Fig. 14 : «France», dessin collé en tête d'un exemplaire d'une édition originale du tome 1 des Contemplations (Maison de Victor Hugo, MVHL 0821, C Con 1856-1) (C) Maisons de Victor Hugo/Roger-Viollet

45. Frances A. Yates, L'Art de la mémoire, Paris, Gallimard, 1975. 46. «À celle qui est restée en France», Les Contemplations; Poésie II, Laffont, p. 555.

47. Reproduits dans CFL, t. XVIII, $n^{\circ} 773$ et 875. 


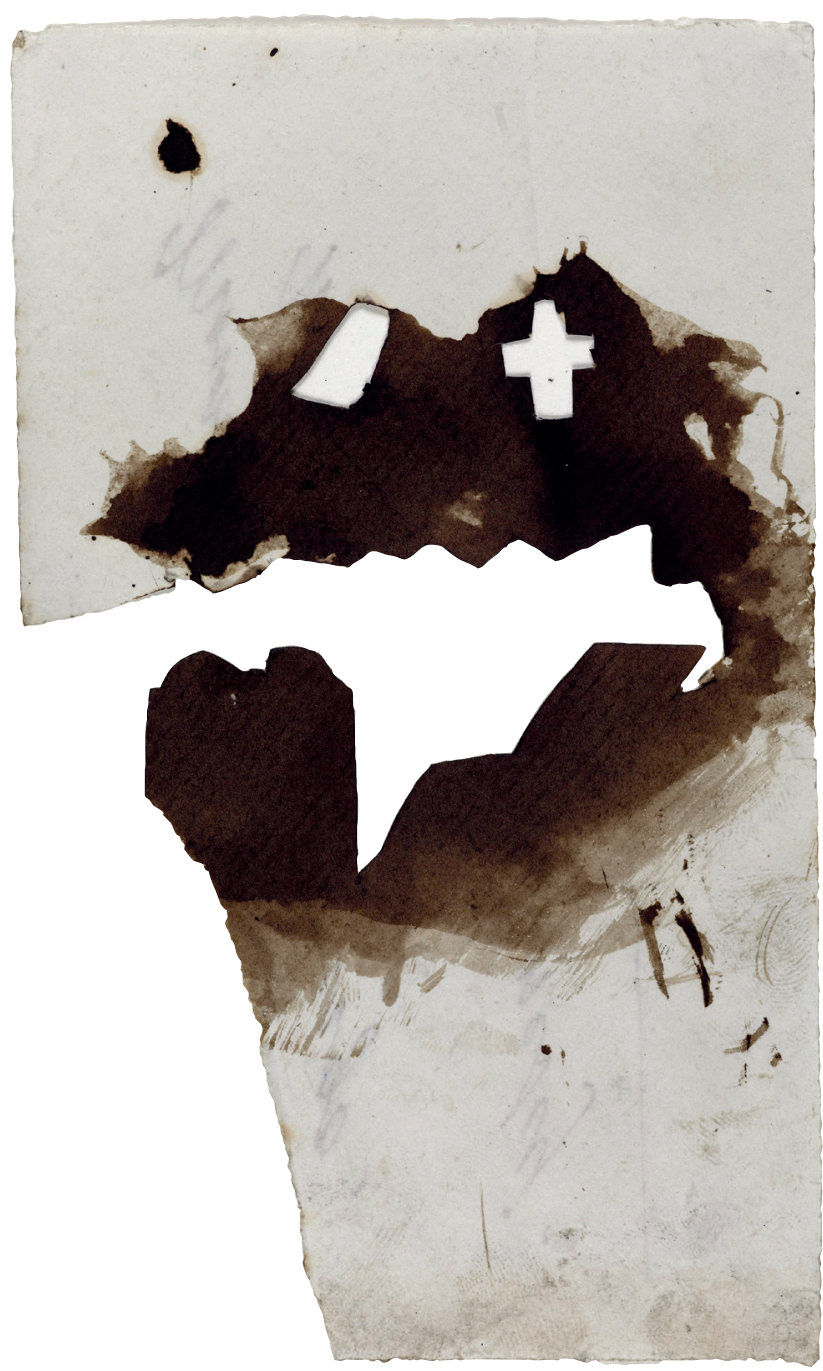

Fig. 15 : «Papier découpé avec une croix ou Étude pour la tombe de Léopoldine», 1858; technique mixte (découpage, lavis brun) (Paris, Maison de Victor Hugo, MVH D 20125 1)

(C) Maisons de Victor Hugo/Roger-Viollet qui la détache de la noirceur de l'encre, retrouvant à sa manière l'antithèse du «noir chemin» fait par le poète et de «ce marbre» qu'il contemple. Le motif est engendré par une forme qui a ensuite été ôtée et dont ne subsiste que l'empreinte.

Le paradoxe de la marche chez Victor Hugo, et que reflète en partie la pratique du dessin, tient sans doute à cette tension entre le réel et le virtuel. Un passage rendu plus sensible encore par les circonstances de la proscription et qui n'avait pas échappé à Baudelaire lorsqu'il écrivait dans son article de 1861 :

Quand aujourd'hui nous parcourons les poésies récentes de Victor Hugo, nous voyons que tel il était, tel il est resté : un promeneur pensif, un homme solitaire mais enthousiaste de la vie, un esprit rêveur et interrogateur. [...] autrefois, il rôdait solitaire dans des lieux bouillonnant de vie humaine; aujourd'hui, il marche dans des solitudes peuplées par sa pensée. [...] Mais là-bas comme ici, toujours il nous apparaît comme la statue de la Méditation qui marche ${ }^{48}$.

«Des solitudes peuplées par sa pensée»: l'hypotypose n'est sans doute pas qu'une figure de style, pas plus que le pochoir une simple fantaisie technique. Ce sont des dominantes énonciatives de l'exil. Manifestations l'une et l'autre de ce qui est absent, trace spectrale de ce qui fait défaut. En un mot, une survivance.

48. Charles Baudelaire, art. cité, loc. cit., p. 130. 
DELPHINE GLEIZES est maître de conférences HDR en littérature française du XIXe siècle à l'université Lumière Lyon 2 et membre de l'UMR 5317 IHRIM. Elle travaille sur l'œuvre littéraire et graphique de Victor Hugo mais ses recherches portent plus largement sur les rapports entre littérature et culture visuelle. Elle a publié L'Euvre de Victor Hugo à l'écran (Québec, PU Laval, 2005), codirigé, avec Sarga Moussa, Juliette Récamier dans les arts et la littérature (Hermann, 2011), et édité avec Denis Reynaud l'anthologie Machines à voir. Pour une histoire du regard instrumenté (XVIIe-XIXe siècles) (PUL, 2017).

Delphine.Gleizes@univ-lyon2.fr

\section{En marchant, en dessinant}

La marche porte Victor Hugo bien au-delà de l'exercice romantique du Voyage pittoresque, même si récits de voyage et carnets hugoliens souscrivent souvent aux codes du genre. La marche apparaît comme l'élément dynamique d'une genèse dont le pas du marcheur, dans son amplitude, serait comme le principe inchoatif. Constitutive d'un rapport au monde, elle est pour Hugo parcours sensible et effort spirituel; elle confère à la main qui écrit, mais aussi à la main qui dessine une charge cinétique dont cet article tente de cerner les différentes valeurs et variations. Cependant, si le dessin peut traduire la présence d'un corps en mouvement ainsi que la façon dont le visible a d'ores et déjà été configuré par l'expérience de la marche, il trahit aussi parfois la façon dont ce même corps s'absente, question avivée encore par l'expérience de l'exil.

Walking takes Victor Hugo far beyond the romantic exercise of the picturesque Journey, even if his travel writings and notebooks often follow the codes of this genre. Walking appears to be the dynamic element of a genesis in which the walker's step, in its breadth, would be a sort of inchoative principle. Constituent of a relationship to the world, for Hugo it is a progress of the senses and a spiritual effort. It bestows on the hand that writes, but also on the hand that draws, a kinetic task of which this article tries to outline the various shades and variations. However, if a drawing can express the presence of a moving body as well as the way the visible has already been shaped by the experience of walking, it also sometimes reveals the way this same body is absent, a matter accentuated by the experience of exile.

Sein Weg führt Victor Hugo weit über die romantische Übung der „Voyage pittoresque“ hinaus, auch wenn die Reiseberichte und Notizbücher Hugos oft in ihren Merkmalen zu jener Gattung passen. Der Weg erscheint als dynamisches Element einer Genese, wo der Schritt des Voranschreitenden in seiner Größe als das inchoative Prinzip fungieren würde. Konstitutiv für ein bestimmtes Verhältnis zur Welt, ist jener Weg für Hugo zugleich Einfühlung und geistige Anstrengung; er überträgt sich auf die schreibende, aber auch auf die zeichnende Hand, die eine kinetische Aufgabe wahrnimmt, von der der vorliegende Artikel die verschiedenen Bedeutungen und Variationen zu umgrenzen versucht. Jedoch, wenn die Zeichnung die Präsenz eines Körpers in Bewegung übertragen kann, so wie das Sichtbare sich immer schon als Erfahrung des Gehens niederschlägt, so verrät sie doch auch manchmal die Art und Weise, in der sich derselbe Körper absentiert - eine Frage, die zusätzlich durch die Erfahrung des Exils an Lebhaftigkeit gewinnt.
La caminata transporta a Víctor Hugo mucho más allá del ejercicio romántico del "viaje pintoresco", aún si sus relatos de viaje y sus libretas adhieren a menudo a los códigos del género. La caminata aparece como el elemento dinámico de una génesis en la cual el paso del caminante, en su amplitud, constituiría algo así como el principio incoativo. Constitutiva de una relación con el mundo, la caminata es para Hugo recorrido sensible y esfuerzo espiritual, que le otorga a la mano que escribe, y también a la que dibuja, una carga cinética, cuyos valores y variaciones este artículo intentará identificar. Sin embargo, si el dibujo puede traducir tanto la presencia de un cuerpo en movimiento, como la manera con que lo visible ha sido ya configurado por la experiencia de la caminata, a veces también revela la manera en que ese cuerpo se ausenta problemática que le experiencia del exilio intensificará todavía más.

A marcha transporta Victor Hugo para além do exercício romântico da Viagem pitoresca, mesmo quando narrativas e livros de apontamentos parecem inscrever-se nos códigos do género. A marcha aparece como elemento dinâmico numa gênese em que a passada do caminhante, na sua amplitude, seria o princípio incoativo. Constitutiva da sua relação com o mundo, a marcha é para Hugo um percurso dos sentidos e um esforço espiritual; ela confere à mão que escreve, e também que desenha, uma carga cinética de que este artigo tenta identificar os valores e as variações. No entanto, se o desenho pode traduzir a presença de um corpo em movimento, ou a maneira como o visível é configurado pela experiência da marcha, ele pode também trair o modo como esse mesmo corpo está ausente, aspecto exacerbado por experiências de exílio.

Il camminare porta Victor Hugo ben oltre l'esercizio romantico del Viaggio pittoresco, anche se i suoi racconti di viaggio e i diari sottostanno spesso ai codici del genere. Il camminare appare come l'elemento dinamico di una genesi della quale il passo del marciatore, nella sua ampiezza, sarebbe quasi il principio incoativo. Costitutivo di un rapporto con il mondo, esso è per Hugo percorso sensibile e sforzo spirituale, che conferisce alla mano che scrive, ma anche a quella che disegna, una carica cinetica di cui quest'articolo tenta di inquadrare i diversi valori e mutamenti. Tuttavia, se il disegno può tradurre la presenza di un corpo in movimento, così come il modo in cui il visibile viene fin da subito configurato dall'esperienza del camminare, esso tradisce anche, a volte, il modo in cui questo stesso corpo si assenta, questione che l'esperienza dell'esilio riaccende ancor di più. 